\title{
Simultaneous pit generation and visualization of pit topography using combined atomic force-scanning electrochemical microscopy
}

\author{
J. Izquierdo ${ }^{\mathrm{a}}$, A. Eifert ${ }^{\mathrm{b}}$, R.M. Souto ${ }^{\mathrm{a}}$, C. Kranz ${ }^{\mathrm{b}}$ \\ ${ }^{a}$ Department of Chemistry, University of La Laguna, P.O. Box 456, E-38200 La Laguna, Tenerife, \\ Canary Islands, Spain \\ ${ }^{b}$ Institute of Analytical and Bioanalytical Chemistry, University of Ulm, Albert-Einstein-Allee 11, D- \\ 89081 Ulm, Germany
}

\begin{abstract}
Combined atomic force microscopy - scanning electrochemical microscopy (AFM-SECM) is for the first time used to generate single corrosion pits on passivating iron surfaces in the micrometer range. The AFM-SECM probe locally generates nitric acid during the oxidation of nitrite ions with the release of protons at selected sites on the surface of the otherwise passive metal. High confinement of passive film breakdown is achieved from the combination of a small probe size and the inhibiting properties of non-reacted nitrite ions on the surrounding passivated surface. Simultaneous visualization of pit nucleation and propagation can be obtained in the same solution without changing the probe by AFM.
\end{abstract}

Keywords: Localized corrosion; Single pit nucleation; Iron; Combined AFM-SECM; Imaging 


\section{Introduction}

Pitting corrosion occurs at many metallic materials in highly localized spots. For better understanding and preventing the involved mechanisms, techniques that provide real time information with high spatial resolution during pit monitoring are a prerequisite. However, this is challenging if homogeneous substrates are undergoing pitting corrosion due to the fast and mainly random characteristics of the pitting distribution as pits have to be already nucleated in order to be detected. Aiming to overcome this limitation, one strategy consists in locally modifying the electrolyte in close proximity to a single desired spot of the passive surface in order to induce the nucleation of a corrosion pit. Several research groups explored the potential of SECM studying pit corrosion with high (electro)chemical resolution. Despite their different methodologies, most of them rely in the localized generation of chloride ions at the SECM tip when located in the proximity of the investigated substrate [1-8]. As the probe is close enough to build a thin electrolyte volume confined between the SECM probe and the sample in which a sufficiently high concentration of this aggressive anion can be generated for the nucleation of a single pit. Chloride ions result from some redox reaction occurring at the tip, whereas the electrical condition of the substrate was separately controlled using a bipotentiostat. This procedure has been successfully employed to produce corrosion pits on iron [2-4,6,7] and stainless steels [1,8], copper [3], and aluminum [1] samples. The dimensions of the generated pits as well as the control for their location were limited by the dimensions of the disc UMEs employed to build a sufficiently high local concentration of chloride ions. As result, pits with diameters ranging several tens to hundreds micrometers were so far obtained. Generation of an aggressive species by redox conversion at the SECM tip was also employed to produce corrosion pits of similar dimensions through alumina layers [9]. In this case, water electrolysis generated high local concentrations of protons (“acid attack”) or hydroxyl ions ("base attack").

Smaller single pits on stainless steel were obtained by direct mode SECM using a UME with $5 \mu \mathrm{m}$ in diameter $[10,11]$. In direct mode a current can be forced to flow between the substrate and the microelectrode tip operating as the working and auxiliary electrodes, respectively. The UME was positioned in shear-force mode and imaging of the corrosion pits was obtained in alternating current (AC) mode SECM; however based on the size of the SECM probe, high resolution imaging of the topology could not been achieved. Due to the smaller tip-to-sample distance in shear-force mode, breakdown of the passive layer on the metal was mostly confined below the tip resulting in corrosion pit with several micrometers in dimension. Since this method does not require the local built-up of a concentrated electrolyte between the tip and the substrate, smaller tips down to a few micrometers 
diameter could be employed [10].

In this contribution we report a new methodology for single pit generation on passive metals based on combined AFM-SECM [12], effectively allowing both controlled pit nucleation and in situ high-resolution imaging of pit formation to be accomplished in the same experiment. AFM-SECM has been used in corrosion science to investigate localized corrosion processes on aluminum alloy AA1050 [13] and also for erosion experiments dissolving the (010) surface of potassium ferrocyanide trihydrate [14]. The electroactive area of the combined AFM-SECM probes is located as a frame electrode above the apex of a non-conductive silicon nitride probe, thus allowing simultaneous electrochemical and topographical imaging. Furthermore, redox species are used that can both operate as corrosion inhibitor, thus enhancing the stability of the passive films formed on the metal, and which serves as the source of the aggressive chemical agent responsible for passive layer breakdown, as result of its electrochemical conversion at the AFM tip-integrated electrode. In this way, in a very confined volume, nitric acid is generated which promotes the surface film attack. Borgwarth and Heinze used nitrite oxidation introducing the concept of "chemical lens" by adding scavenger molecules to solution for controlled electrodeposition using SECM [15]. The high spatial resolution of AFM allows the simultaneous imaging of the generated pits, as well as to follow the evolution of the nucleated features on the otherwise passive metallic substrate.

\section{Experimental}

Experiments were performed on 99.5\% purity iron plates (Goodfellow Materials, Cambridge, UK) of dimensions $2.5 \mathrm{~cm} \times 2.5 \mathrm{~cm}$ and thickness $1 \mathrm{~mm}$. The surface was ground to a 4000 finish, followed by polishing with $0.05 \mu \mathrm{m}$ alumina slurry. The resulting surface was degreased with ethanol in an ultrasonic bath, subsequently rinsed with ultra-pure deionized water and allowed to dry under argon gas stream. Solutions were prepared using analytical grade chemicals and Millipore water (18.0 $\mathrm{M} \Omega \mathrm{cm})$. Experiments were performed in naturally aerated solutions at ambient temperature (ca. $20^{\circ} \mathrm{C}$ ).

Integrated AFM-SECM probes were fabricated following a procedure described elsewhere [16]. In brief, commercially available silicon nitride cantilevers were first coated with a $100 \mathrm{~nm}$ thick gold layer, followed by an insulation step with silicon nitride using chemical vapor deposition. Focus ion beam (FIB) was employed for precisely exposing a frame-shaped electrode and re-shaping the very end of the AFM tip to form the thorn for high-resolution topography measurement. Figure 1 shows a scanning electron micrograph and scheme of the dual probe presenting a square gold electrode with a length of $1.07 \mu \mathrm{m}$, and a thorn of $0.46 \mu \mathrm{m}$ in height. The electrochemical response 
of the AFM-SECM probe was characterized in deoxygenated $10 \mathrm{mM}$ potassium hexaaminruthenium (III) solution using $0.1 \mathrm{M} \mathrm{KCl}$ as supporting electrolyte.

The AFM-SECM set-up consisted of an atomic force microscope equipped with a SECM module and a picostat potentiostat from Keysight Technologies (Chandler, AZ, USA). The SECM module included a specialized nosecone with built-in operation amplifier and all the necessary connections for SECM operation. The iron substrate was carefully mounted in the AFM liquid cell, using an O-ring for exposing a circular area of $1.75 \mathrm{~cm}$ in diameter. The contained volume of electrolyte was approximately $1 \mathrm{~mL}$. A ring-shaped counter electrode made of platinum, and a pseudo-reference electrode made of chlorinated silver wire completed the three-electrode cell with the AFM-SECM probe as working electrode. Potential of the $\mathrm{Ag} / \mathrm{AgCl}$ wire pseudo-reference electrode resulted in $+48 \mathrm{mV}$ vs. $\mathrm{Ag} / \mathrm{AgCl}$ (sat.) reference electrode in the test solution, consisting of $0.5 \mathrm{M} \mathrm{NaCl}+0.1 \mathrm{M} \mathrm{NaNO}_{2}$. For the sake of simplicity, all potential values are referred to the $\mathrm{Ag} / \mathrm{AgCl}$ (sat.) reference electrode. Throughout the presented experiments, the iron samples were left unbiased, effectively attaining their spontaneous OCP values in the test solutions.

The mass transport towards the combined AFM-SECM probes was simulated with using ComSol 4.1 using a three dimensional virtual replica of the combined probe. For the numerical calculations, only diffusion towards the electrode was taken into account. For the numerical calculation, a diffusion coefficient for nitrite of $D=1.912 \times 10^{-5} \mathrm{~cm}^{2} \mathrm{~s}^{-1}$ [17] was used. Detailed description about the simulation can be found elsewhere [18].

\section{Results and discussion}

A localized pit induction strategy was developed for the generation of single pits on spontaneously passivated iron samples induced by the AFM tip-integrated electrode, which is schematically depicted in Figure 1. In addition to chloride ions, which are an aggressive species promoting pitting corrosion of iron and its alloys, the solution contained also nitrite ions. The latter are known as corrosion inhibitor for iron, effectively promoting the formation of protective oxide layers on the metal, which mechanisms have been discussed in literature [19,20]. In addition, nitrite ions can be oxidized to nitrate at the AFM-SECM probe forming locally nitric acid. Cyclic voltammograms recorded at gold disc UME (25 $\mu \mathrm{m}$ dia.) immersed in $0.1 \mathrm{M} \mathrm{NaNO}_{2}+0.5 \mathrm{M} \mathrm{NaCl}$ solution revealed that the onset of nitrite oxidation is observed at potential values more positive than approximately $+0.7 \mathrm{~V}$ vs. $\mathrm{Ag} / \mathrm{AgCl}$ (sat.). The diffusion-limited potential range occurs beyond +0.9 V vs. Ag/AgCl (sat.).

Accordingly, the potential of the AFM-SECM probe was thus set to a value at which the 
diffusion-limited oxidation of nitrite to nitrate occurred in accordance with [21,22]:

$$
\mathrm{NO}_{2}{ }^{-}+\mathrm{H}_{2} \mathrm{O} \rightarrow \mathrm{NO}_{3}{ }^{-}+2 \mathrm{H}^{+}
$$

The probe releases protons into the solution as by-product of the reaction. These protons originate a strong acidic environment in the small electrolyte volume comprised between the AFM-SECM probe and the iron substrate. The passivating effect of nitrite ions is thus ensured on the exposed iron substrate except in the area below the AFM-SECM probe due to the highly localized generation of the aggressive species. Nitrite oxidation at the probe-integrated electrode generates nitric acid locally, and this local acidification combined with the high bulk chloride content lead to a local rapid attack of the surface, and pit formation below the AFM-SECM probe. Besides the improved resolution in respect to the generated pits and exact control of pit formation, the combination of SECM and AFM allowed simultaneous imaging of the generated pit with high resolution, without requiring the change of probe or test solution. 3D-AFM images of the iron surface obtained in contact mode just before (Fig. 2A) and after (Fig. 2B) locally inducing the nucleation of a corrosion pit on the iron surface are shown in Figure 2. A circular-shaped pit of approximately $4 \mu \mathrm{m}$ diameter, located in the center of a shallow circular depression of ca. $8 \mu \mathrm{m}$ diameter (see Fig. 2D), was generated by applying a bias of $+0.95 \mathrm{~V}$ vs. $\mathrm{Ag} / \mathrm{AgCl}$ (sat.) at the AFM tip-integrated electrode, while scanning an area of $3 \times 3 \mu^{2}$. Besides improved spatial resolution and exact control of pit formation, simultaneous imaging of the generated pit is performed by the AFM tip centered with respect to the frame electrode, without requiring the change of probe or solution.

Figure 3A shows the concentration profile at the AFM-SECM probe in nitrite solution. It demonstrates that the acidification effect extends laterally beyond the dimensions of the gold frame integrated in the AFM tip. As result, bigger holes should be expected when scanning the probe during pit formation compared to a static operation for the generation of the pit. Indeed, Figure 3B shows a pit generated while the scan movement was stopped and the AFM-SECM was biased for 50 seconds at. $+0.95 \mathrm{~V}$ vs. $\mathrm{Ag} / \mathrm{AgCl}$ (sat.). As clearly visible in the topography image $\left(6 \mathrm{x} 6 \mu \mathrm{m}^{2}\right)$, a shallower smaller pit was created.

\section{Conclusions}

The results presented here demonstrate that combined AFM-SECM can be employed to generate single corrosion pits while the pit topography can be imaged consecutively without change of the probe or solution.

The enhanced spatial resolution was achieved by the choice of nitrite ions as redox mediator for SECM operation in the tip generation/substrate collection (TG/SC) operation mode. This chemical species present the unique characteristics of acting as corrosion inhibitor for iron in its 
reduced state, while its oxidation releases protons that effectively modify the electrolyte environment at will, with the result of pit induction in a chloride-containing solution.

In addition, the reshaped AFM tip with a similar curve radius as commercially available probes, provides in situ topographical imaging of the surface at the location of pit induction, and can be employed to continue imaging the evolution of a corrosion pit once nucleated into its propagation or eventual repassivation stages.

We regard the methods described in this contribution to offer a powerful means for the investigation of the early stages of passivity breakdown and localized corrosion.

\section{Acknowledgments:}

A grant awarded to JIP by the Deutscher Akademischer Austauschdienst (Berlin, Germany) to conduct a research stay at the University of Ulm is gratefully acknowledged. The Focused Ion Beam Center UUlm supported by FEI Company (Eindhoven, Netherlands), the German Science Foundation (INST40/385-F1UG), and the Struktur- und Innovationsfonds Baden-Württemberg are acknowledged for support of this study.

\section{References:}

[1] D.O. Wipf, Coll. Surf. A 93 (1994) 251.

[2] J.W. Still, D.O. Wipf, J. Electrochem. Soc. 144 (1997) 2657.

[3] K. Fushimi, K. Azumi, M. Seo, J. Electrochem. Soc. 147 (2000) 552.

[4] K. Fushimi, M. Seo, J. Electrochem. Soc. 148 (2001) B450.

[5] F. Falkenberg, K. Fushimi, M. Seo, Corros. Sci. 45 (2003) 2657.

[6] C. Gabrielli, S. Joiret, M. Keddam, H. Perrot, N. Portail, P. Rousseau, V. Vivier, J. Electrochem. Soc. 153 (2006) B68.

[7] C. Gabrielli, S. Joiret, M. Keddam, H. Perrot, N. Portail, P. Rousseau, V. Vivier, Electrochim. Acta 52 (2007) 7706.

[8] N. Aouina, F. Balbaud-Célérier, F. Huet, S. Joiret, H. Perrot, F. Rouillard, V. Vivier, Electrochim. Acta 56 (2011) 8589.

[9] D. Battistel, S. Daniele, R. Gerbasi, M.A. Baldo, Thin Solid Films 518 (2010) 3625.

[10] K. Eckhard, M. Etienne, A. Schulte, W. Schuhmann, Electrochem. Commun. 9 (2007) 1793.

[11] K. Eckhard, T. Erichsen, M. Stratmann, W. Schuhmann, Chem. Eur. J. 14 (2008) 3968.

[12] C. Kranz, Analyst 139 (2014) 336. 
[13] A. Davoodi, J. Pan, C. Leygraf, and S. Norgren, Electrochem. Solid-State Lett. 8 (2005) B21.

[14] C. E. Jones, J. V. Macpherson, P. R. Unwin, J. Phys. Chem. B. 104 (2000) 2351.

[15] K. Borgwarth, J. Heinze, J. Electrochem. Soc. 146 (1999) 3285.

[16] C. Kranz, G. Friedbacher, B. Mizaikoff, A. Lugstein, J. Smoliner, E. Bertagnolli, Anal. Chem. 73 (2001) 2491.

[17] Handbook of Chemistry and Physics, 84th Edition, (ed., D. R. Lide), CRC PRESS, (2003-2004).

[18]A. Eifert, W. Smirnov, S. Frittmann, C. E. Nebel, B. Mizaikoff, C. Kranz, Electrochem. Commun. 25 (2012) 30.

[19] M. Reffass, R. Sabot, M. Jeannin, C. Berziou, Ph. Refait, Electrochim. Acta 52 (2007) 7599.

[20] D.Y. Lee, W.C. Kim, J.G. Kim, Corros. Sci. 64 (2012) 105.

[21] J. Ufheil, F.M. Boldt, M. Börsch, K. Borgwarth, J. Heinze, Bioelectrochem. 52 (2000) 103.

[22] V. Radtke, C. Heß, R.M. Souto, J. Heinze, Z. Phys. Chem. 220 (2006) 393.

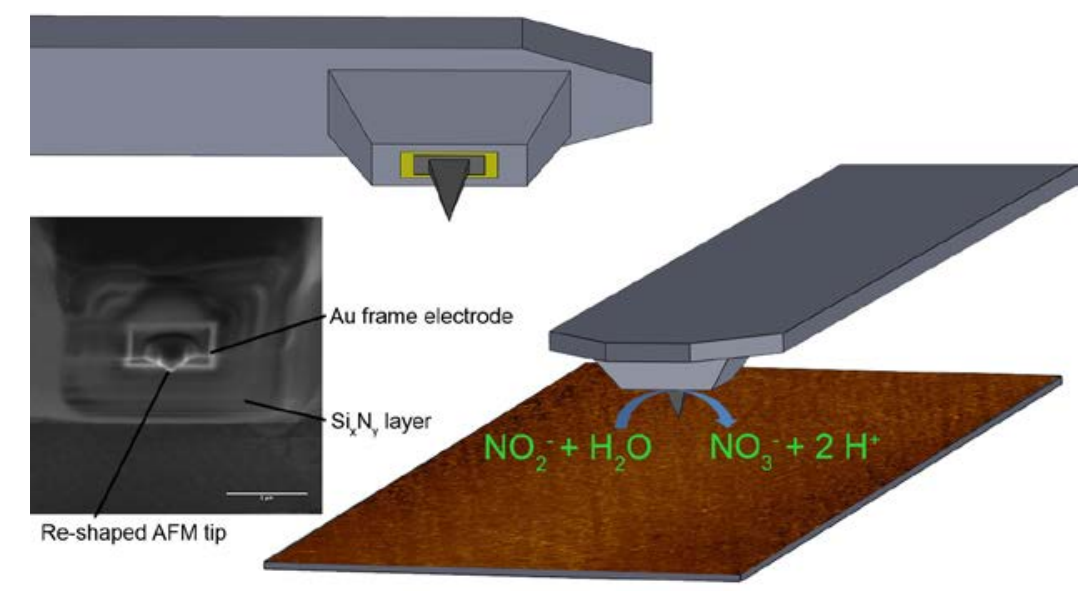

Figure 1

Scheme of pit induction on an iron substrate in tip generation/substrate collection (TG/SC) mode. Scheme of AFM-SECM probe and SEM image of the combined probe. Nitrite is oxidized to nitrate at the AFM-SECM probe and protons are generated as by-product. In the gap between the probe and the surface a highly acid chloride-containing electrolyte is produced, resulting in the localized breakdown of the passive oxide layer present on the metal. 
A)
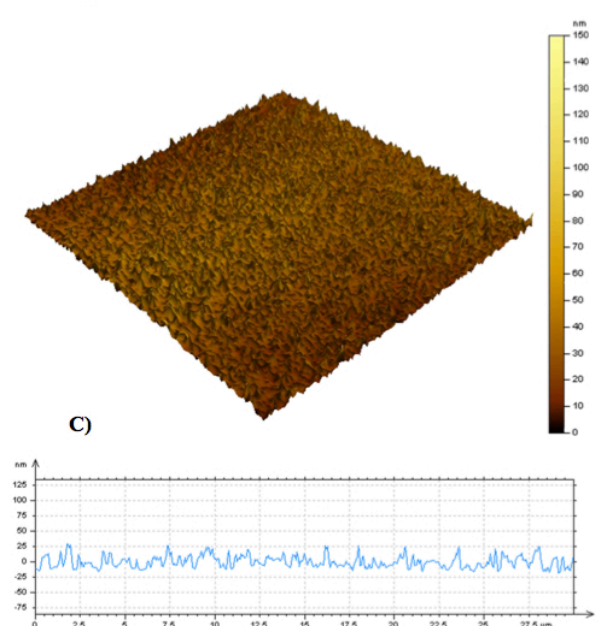

B)

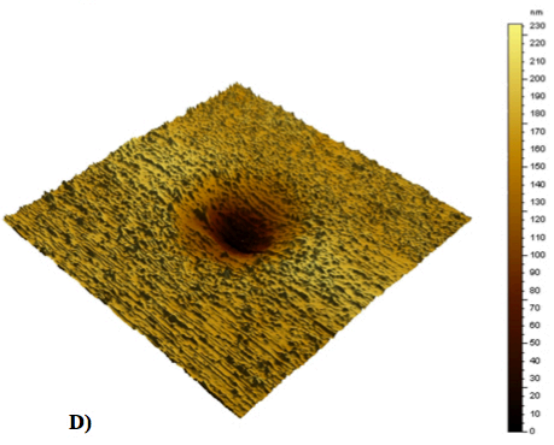

D)

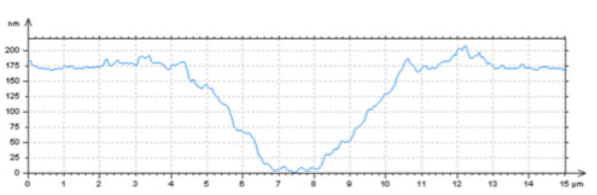

Figure 2

3D AFM topography of the iron surface obtained in contact mode in nitrite solution recorded with a combined AFM-SECM probe. Scans were performed (A) before, and (B) after inducing the localized breakdown of the iron surface by setting the AFM tip-integrated electrode at $+0.95 \mathrm{~V}$ vs. Ag/AgCl (sat.) while scanning a $3 \times 3 \mu \mathrm{m}^{2}$ area of the surface in the center of the sample.
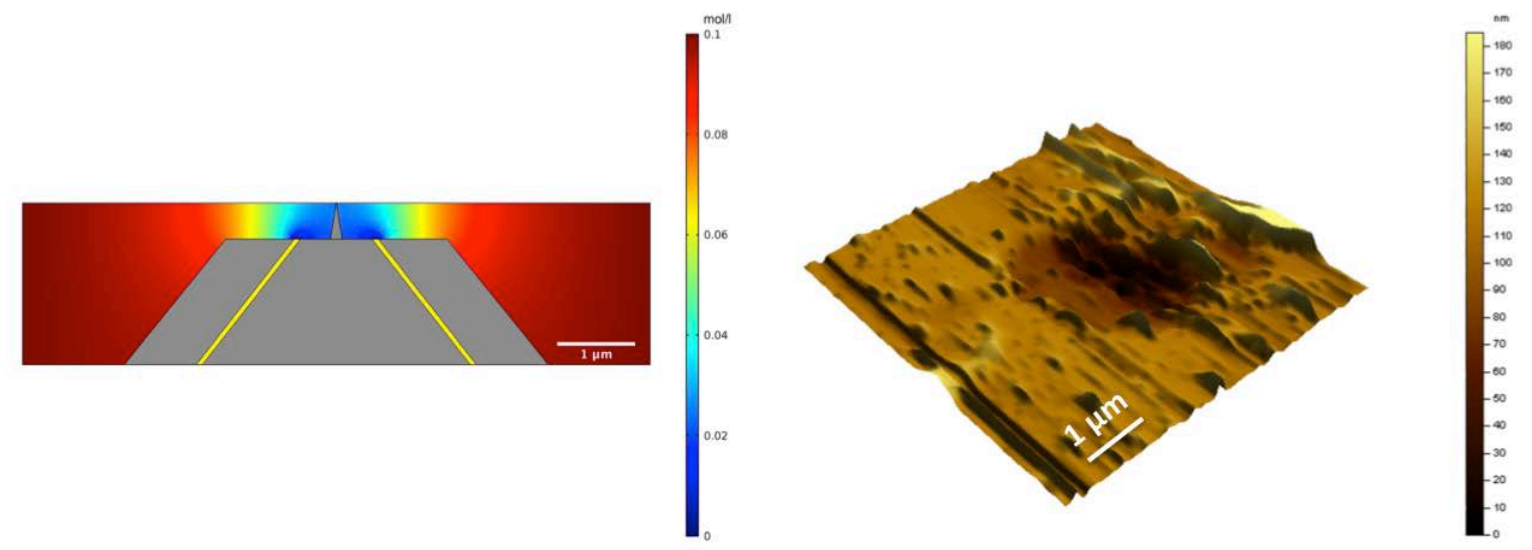

A

B

Figure 3

(A) Simulated concentration profile in nitrite solution at an AFM tip-integrated electrode with an electrode edge length of $1.07 \mu \mathrm{m}$. (B) 3D AFM topography of a stationary pit experiment for $50 \mathrm{sec}$ recorded in contact mode. 